\title{
Revision Anterior Cruciate Ligament Reconstruction with Contralateral Hamstring Tendon Grafts: 6 Years Follow-Up
}

\author{
Claudio Legnani ${ }^{1}$ Stefania Zini ${ }^{2}$ Enrico Borgo ${ }^{3}$ Alberto Ventura ${ }^{3}$
}

${ }^{1}$ IRCCS Galeazzi Orthopaedic Institute, Milan, Italy

${ }^{2}$ Scuola di Specializzazione in Ortopedia e Traumatologia, Policlinico

Universitario P. Giaccone, Palermo, Italy

${ }^{3}$ Sports Traumatology and Minimally Invasive Articular Surgery

Orthopaedic Institute, Via Galeazzi 4, 20164 Milan, Italy (e-mail: clegnan@alice.it).

Center, San Siro Clinical Institute, Milan, Italy

Joints 2017;5:17-20.

\begin{abstract}
Keywords

- anterior cruciate ligament

- revision surgery

- contralateral

- hamstring tendons

- return to sports

Purpose The purpose of this study was to retrospectively evaluate the clinical outcome of revision anterior cruciate ligament $(\mathrm{ACL})$ reconstruction with contralateral hamstring tendon autografts, specifically with regard to patient satisfaction, return to preinjury activity level, and postoperative functional outcomes.

Methods Between 2004 and 2011, 23 patients underwent revision ACL reconstruction with contralateral autogenous hamstring tendon grafts and were retrospectively reviewed at an average follow-up of 6.3 years. Subjective and functional evaluations were performed. The Tegner score, Knee Injury and Osteoarthritis Outcome Score (KOOS), and International Knee Documentation Committee (IKDC) Subjective Knee Form were used. Objective evaluation included range of motion, Lachman test, pivot-shift test, and KT-1000 instrumented laxity testing. Wilcoxon test was used to compare the preoperative and follow-up status. Differences with a $p$-value of $<0.05$ were considered statistically significant.

Results No major complications were reported. The mean KOOS significantly increased from a preoperative mean of $62.8 \pm 8.3$ to $85.8 \pm 6.9(p<0.001)$. IKDC subjective score significantly improved from $29.2 \pm 10.4$ to $72.8 \pm 5.2(p<0.001)$. The median Tegner activity score significantly improved from a preoperative mean of 6.5 (range: $4-10$ ) to 7.5 (range: $7-10)(p<0.001)$. Most of the patients increased or returned to the same activity level, with $61 \%$ of the patients returning to cutting and pivoting sports.

Conclusion The use of contralateral hamstring tendon autografts for $A C L$ revision surgery represents a valid option following a failed primary $A C L$ reconstruction and confirms subjective and objective clinical improvement 6 years after surgery. Level of Evidence Level IV, therapeutic case series.
\end{abstract}

\section{Introduction}

The number of revision anterior cruciate ligament (ACL) surgery has risen over the past few years. ${ }^{1}$ Revision ACL surgery is recommended for patients who have symptomatic objective pathological laxity after a failed primary ACL reconstruction.

The choice of graft in revision surgery remains a debatable issue. Autografts constitute a popular choice as many authors recommend autografts both for primary and revision ACL reconstruction. ${ }^{1-6}$ Bone-patellar tendon-bone (BPTB) is frequently used as preferred graft source for ACL reconstruction in case of hamstring failure and vice versa. $^{7}$ To minimize donor-site morbidity and to reduce operative time and incisions, allografts have been gaining in popularity over the past few years. ${ }^{8}$ In addition, contralateral doubled gracilis and semitendinosus tendon (DGST) graft or BPTB has been proposed as a viable option for ACL revision surgery. ${ }^{7,9}$ 
The aim of this study was to evaluate the clinical outcome of ACL revision surgery using the contralateral hamstring tendon autografts. The hypothesis of the study was that contralateral hamstring tendon autograft in ACL revision surgery provides a satisfactory outcome, specifically with regard to patient satisfaction, return to sports and to preinjury activity level, and knee function.

\section{Methods}

\section{Participants}

Between 2004 and 2011, 23 patients underwent revision ACL reconstruction with contralateral autogenous hamstring tendon grafts and were retrospectively reviewed at an average follow-up of 6.3 years. Inclusion criterion was failed primary ACL reconstruction, confirmed by recurrence of giving-way episodes and revealed by positive Lachman and pivot-shift tests. Exclusion criteria were multiligament knee injuries, contralateral instability or other significant knee diseases, severe chondral damage (grade 3 or 4 according to the Outerbridge classification system), and degenerative arthritis (Ahlback grade 3 of 4). - Table 1 shows complete demographic and anthropometric data.

Written informed consent was obtained from all the patients. The local ethic committee approved the study.

\section{Interventions}

Preliminary arthroscopic evaluation was performed to confirm the diagnosis. Patients were excluded from the study when intraoperative findings did not match inclusion criterion.

Hamstring tendon grafts were harvested from the contralateral knee with a tendon stripper through an incision over the pes anserinus on the anteromedial aspect of the tibia and then prepared to form a four-strand graft. Tibial and femoral tunnels were drilled with an arthroscopically assisted transtibial technique. After the remnant of the torn ACL graft was removed, the tibial tunnel was drilled with the aid of a compass guide (Acufex, Smith \& Nephew, Andover, Massachusetts, United States) at a 55-degree angle in the horizontal plane on the tibial plateau. The femoral tunnel was then drilled on the lateral wall of the intercondylar notch with the knee flexed at 90 to 120 degrees, to a depth of $30 \mathrm{~mm}$. Tunnel diameter was matched with the width of the prepared graft. In most cases, tunnels from primary ACL reconstruction were correctly placed and could

Table 1 Patient demographics and anthropometric data

\begin{tabular}{|l|l|}
\hline No. of patients & 23 \\
\hline Gender (male/female) & $14 / 9$ \\
\hline $\begin{array}{l}\text { Mean time from reinjury to surgery }(\mathrm{mo}) \\
\text { (mean } \pm \text { SD) }\end{array}$ & $6.6 \pm 1.2$ \\
\hline Weight $(\mathrm{kg})($ mean $\pm \mathrm{SD})$ & $70.5 \pm 6.9$ \\
\hline Height $(\mathrm{cm})($ mean $\pm \mathrm{SD})$ & $172.7 \pm 6.4$ \\
\hline Age at surgery $(\mathrm{y})($ mean $\pm \mathrm{SD})$ & $26.8 \pm 8.8$ \\
\hline
\end{tabular}

Abbreviation: SD, standard deviation. therefore be reused for revision surgery after accurate redrilling. In those cases in which tunnels were reoriented, care was taken to avoid convergence with the previous tunnels. Then, the graft was fixed proximally with the use of a Tightrope device (Arthrex, Naples, Florida, United States). Distal fixation was achieved through a BioRCI screw (Smith \& Nephew), having a diameter 1 or $2 \mathrm{~mm}$ larger than that of the graft, while the knee was kept at 20 degrees of flexion and the graft under maximal manual tension.

For the first 4 weeks after surgery, walking with partial weight-bearing was allowed with the use of two crutches. Full weight-bearing was allowed as tolerated on the harvested knee. Patients were encouraged to regain proprioception with the use of a balance board and complete knee flexion and extension. Closed kinetic chain exercises were performed for the first 3 months, and thereafter open kinetic chain exercises were started. Swimming and indoor cycling were permitted after 12 weeks, jogging and noncontact sports were permitted after 5 months, and a return to contact sports was allowed after 6 months.

\section{Outcome Measurements}

Patients were evaluated preoperatively and after an average follow-up of 6.3 years (range: 2-8 years). Assessment included Knee Osteoarthritis Outcome Score (KOOS), International Knee Documentation Committee (IKDC) Subjective Knee Form, and Tegner activity level score. Objective examination included instrumented laxity test with KT-1000 arthrometer (MEDmetric Corporation, San Diego, California, United States) under a 134-N anterior tibial load. All patients were evaluated by the same examiner.

\section{Data Analysis}

Data extracted were analyzed using the software SPSS Version 19.0 (SPSS Inc., Chicago, Illinois, United States). Wilcoxon test was used to compare the preoperative and follow-up status. Differences with a $p$-value of $<0.05$ were considered statistically significant.

\section{Results}

No major complications were reported. Subjectively, all patients were satisfied with their revision ACL reconstruction. The mean KOOS significantly increased from a preoperative mean of $62.8 \pm 8.3$ to $85.8 \pm 6.9(p<0.001)$. IKDC subjective score significantly improved from $29.2 \pm 10.4$ to $72.8 \pm 5.2$ $(p<0.001)$. The median Tegner activity score significantly improved from a preoperative mean of 6.5 (range: $4-10$ ) to 7.5 (range: $7-10)(p<0.001)$. Lachman test and pivot-shift test were significantly improved when compared with preoperative status ( $p<0.001)$. The mean KT-1000 value (side-to-side difference) was $1.7 \pm 2.4 \mathrm{~mm}$. Of the patients, 21 (91\%) reported a value equal to or less than $3 \mathrm{~mm}$ compared with the contralateral knee. A detailed overview of the results of overall clinical assessment is shown in - Table 2.

Of $23,17(74 \%)$ increased or returned to the same activity prior to injury and $61 \%$ of the patients returning to cutting and pivoting sports ( - Table 3 ). 
Table 2 Overview of the results of clinical assessment

\begin{tabular}{|l|l|l|l|}
\hline & Preoperative & Postoperative & $p$-Value \\
\hline KOOS (mean \pm SD) & $62.8 \pm 8.3$ & $85.8 \pm 6.9$ & $p<0.001$ \\
\hline IKDC subjective score (mean \pm SD) & $29.2 \pm 10.4$ & $72.8 \pm 5.2$ & $p<0.001$ \\
\hline Tegner activity level score [median (range)] & $6.5(4-10)$ & $7.5(6-10)$ & $p<0.001$ \\
\hline Positive Lachman test $(n[\%])$ & $23(100)$ & $0(0)$ & $p<0.001$ \\
\hline Positive pivot-shift test $(n[\%])$ & $23(100)$ & $2(9)$ & $p<0.001$ \\
\hline KT-1000 (side-to-side difference) $(\mathrm{mm})($ mean \pm SD) & $5.8 \pm 1.8$ & $1.7 \pm 2.4$ & $p<0.001$ \\
\hline
\end{tabular}

Abbreviations: IKDC, International Knee Documentation Committee; KOOS, Knee Injury and Osteoarthritis Outcome Score; SD, standard deviation.

One patient had mild contralateral harvest site symptoms. No subjective loss of motion or strength of the contralateral knee nor long-term significant morbidity at follow-up were reported.

\section{Discussion}

This study shows favorable results for revision ACL reconstruction with contralateral hamstring tendon autografts concerning subjective knee function and knee stability, as well as ability to resume sports activities. Results are comparable to the most satisfactory outcomes reported in the literature. ${ }^{1,7}$

Subjectively, all patients reported that they would have the surgery again and were satisfied compared with their prerevision status. After an average time of 6 years from surgery, mean KOOS was 85.8 , whereas subjective IKDC score was 72.8. None of the patients reported knee instability. Concerning anterior tibial translation, mean side-to-side KT-1000 value significantly improved form preoperative status.

Return to sports following ACL surgery, and the capacity of resuming sporting activities comparable to those engaged prior to the traumatic injury, is a major concern for patients undergoing ACL surgery. ${ }^{10}$ Shelbourne et $\mathrm{al}^{11}$ reported a rate of return to sports ranging from 62 to $74 \%$ in athletes

Table 3 Overview of sports activity preoperatively and at follow-up

\begin{tabular}{|l|l|l|}
\hline & $\begin{array}{l}\text { Preinjury, } \\
\boldsymbol{n}(\%)\end{array}$ & $\begin{array}{l}\text { Post-operative, } \\
\boldsymbol{n}(\%)\end{array}$ \\
\hline Jumping, pivoting, cutting & $16(70)$ & $14(61)$ \\
\hline Swimming, cycling & $7(30)$ & $7(30)$ \\
\hline No sports & $0(0)$ & $2(9)$ \\
\hline Change in sports activity & & \\
\hline Increased level & & $2(9)$ \\
\hline Same level & & $15(65)$ \\
\hline Decreased level & & $4(17)$ \\
\hline No participation & & $2(9)$ \\
\hline
\end{tabular}

who underwent revision ACL reconstruction with a patellar tendon autograft.

In our case series, $74 \%$ of patients were able to return to their preinjury level of sports activity. This result is in line with that of other studies with similar follow-up. ${ }^{12}$ It has been previously demonstrated that improved sports function following ACL revision surgery is obtained when an autograft is used. ${ }^{13}$ Revision ACL reconstruction with DGST contralateral graft may therefore be one possible option in patients with recurrent knee instability following primary repair who wish to return to their preinjury activity level.

Donor-site morbidity represents a major concern in case of tendon harvesting from a healthy knee. In our case series, no complications were observed, and at subjective evaluation, only one patient reported mild contralateral harvest site symptoms.

Limitations of this study include its retrospective nature, its relatively small sample size, and the lack of a control group.

In conclusion, the use of contralateral hamstring tendon autografts is a valid therapeutic option for ACL revision surgery and confirms subjective and objective clinical improvement 6 years after surgery.

\section{References}

1 Kamath GV, Redfern JC, Greis PE, Burks RT. Revision anterior cruciate ligament reconstruction. Am J Sports Med 2011;39(01): 199-217

2 Colosimo AJ, Heidt RS Jr, Traub JA, Carlonas RL. Revision anterior cruciate ligament reconstruction with a reharvested ipsilateral patellar tendon. Am J Sports Med 2001;29(06):746-750

3 Kartus J, Stener S, Lindahl S, Eriksson BI, Karlsson J. Ipsi- or contralateral patellar tendon graft in anterior cruciate ligament revision surgery. A comparison of two methods. Am J Sports Med 1998;26(04):499-504

4 Noyes FR, Barber-Westin SD. Revision anterior cruciate surgery with use of bone-patellar tendon-bone autogenous grafts. J Bone Joint Surg Am 2001;83-A(08):1131-1143

5 Garofalo R, Djahangiri A, Siegrist O. Revision anterior cruciate ligament reconstruction with quadriceps tendon-patellar bone autograft. Arthroscopy 2006;22(02):205-214

6 Noyes FR, Barber-Westin SD. Anterior cruciate ligament revision reconstruction: results using a quadriceps tendon-patellar bone autograft. Am J Sports Med 2006;34(04):553-564 
20 ACL Revision with Contralateral Hamstrings Legnani et al.

7 Ferretti A, Monaco E, Caperna L, Palma T, Conteduca F. Revision ACL reconstruction using contralateral hamstrings. Knee Surg Sports Traumatol Arthrosc 2013;21(03):690-695

8 Johnson DL, Swenson TM, Irrgang JJ, Fu FH, Harner CD. Revision anterior cruciate ligament surgery: experience from Pittsburgh. Clin Orthop Relat Res 1996;325:100-109

9 Rubinstein RA Jr, Shelbourne KD, VanMeter CD, McCarroll JC, Rettig AC. Isolated autogenous bone-patellar tendon-bone graft site morbidity. Am J Sports Med 1994;22(03):324-327

10 Giron F, Losco M, Giannini L, Buzzi R. Femoral tunnel in revision anterior cruciate ligament reconstruction. Joints 2014;1(03):126-129
11 Shelbourne KD, Benner RW, Gray T. Return to sports and subsequent injury rates after revision anterior cruciate ligament reconstruction with patellar tendon autograft. Am J Sports Med 2014;42(06):1395-1400

12 Peterson RK, Shelton WR, Bomboy AL. Allograft versus autograft patellar tendon anterior cruciate ligament reconstruction: A 5-year follow-up. Arthroscopy 2001;17(01):9-13

13 MARS Group; MARS Group. Effect of graft choice on the outcome of revision anterior cruciate ligament reconstruction in the Multicenter ACL Revision Study (MARS) Cohort. Am J Sports Med 2014; 42(10):2301-2310 